\title{
One-dimensional Models for Cell Feeding in Micro-channels
}

\author{
I. Nesteruk \\ Institute of Hydromechanics, NASU \\ NTUU "Igor Sykorsky Kyiv Polytechnic Institute" \\ Kyiv, Ukraine \\ inesteruk@yahoo.com
}

\author{
R. Visone, A. Redaelli \\ Department of Electronics, Information and Bioengineering \\ Politecnico di Milano \\ Milano, Italy \\ roberta.visone@polimi.it, alberto.redaelli@polimi.it
}

\begin{abstract}
Lab-on-Chip technology is an emerging tool to obtain, culture and study different kinds of tissue models. In particular, concerning cardiac microtissues, it could be exploited to investigate cellular behavior in response to biomimetic stimulation (e.g., electrical and/or mechanical actuations).Cell culture in microfluidic chip, usually last several days. During this period of time, cells need feeding to stay alive, proliferate and organize. Different configurations and sizes of microchip features can cause non-uniform distribution of nutrients and wastes on chip, which must be taken into account in studying the response of cells to electrical and/or mechanical stimulations. A simple onedimensional model of diffusion was used to simulate the concentration of a nutrient and a waste in a previously developed microfluidic chip. For quasi-steady processes, analytical solutions were obtained and analyzed.
\end{abstract}

Keywords-Organ-on-Chip technology;cell culture;microfluidic channels; diffusion equations; analytical solutions.

\section{INTRODUCTION}

The Organ-on-a-Chip technology has recently emerged as fundamental tool in biology to advance the study of tissue development and organ physiology/pathology [1]. By mimicking the complexity of native cellular milieu, these microfluidic cell culture platforms produce levels of tissue functionality not achievable with conventional culture systems [2], opening the opportunity to investigate cell behavior in response to different biomimetic conditions (e.g., mechanical and electrical stimulation of cardiac cells [3], [4]).Miniaturization also allows for gaining a superior control over the local cellular microenvironment and reduces the use of reagents and cells [5]. In this way highly dense constructs like cardiac microtissues can be generated without the need of tissue vascularization [6]. To generate functional cardiac microtissue, the cell culture in chip is performed for several days [6]. During this period, cells need to be constantly fed to be alive, to proliferate and to organize. Consequently, cell culture media containing nutrients is refreshed daily. During this relatively long time, the concentrations of nutrients and wastes changes, influencing the metabolic processes in cells. Different configurations and sizes of microchip features can cause non-uniform distribution of nutrients and wastes within the chip. Mathematical models could be optimal tools to investigate these phenomena and give insights to better interpret the response of cells to electrical and/or mechanical stimulations. In this contest some mathematical models of oxygen and nutrients transfer in cell culture micro-channels were previously developed in [7], [8]. In this paper, we will use a simple one-dimensional model of diffusion (see e.g.,[9]) in order to simulate the concentration of a nutrients and wastes within a previously developed microfluidic chip, designed to generate functional cardiac tissue providing cells with biomimetic mechanical stimulation [6]. It was shown that the processes of changes in concentration are quasi-steady. This fact simplifies the analysis and allows obtaining analytical solutions

\section{MATERIALS AND METHODS}

\section{A. Chip configuration and dimensions}

The microfluidic chip comprises three functional element (see Fig.1a): i) the cell culture chamber, where 3D microtissue are generated in the central region of the channel, lined by culture medium, ii) a thin PDMS membrane and iii) the actuation chamber, which can be pressurized to deform the membrane and to apply cyclic mechanical deformation to the above positioned 3D microtissue.

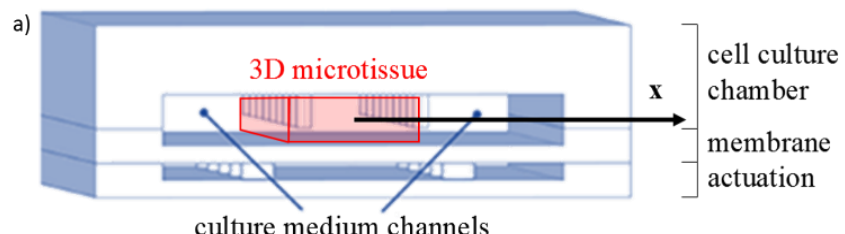

b)

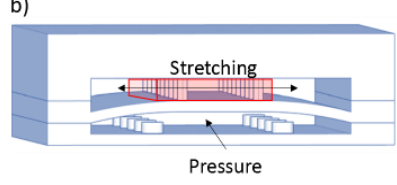

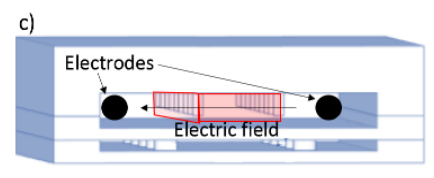

Fig. 1. a) Schematic representation of the microfluidic chip depicting the cell culture chamber (upper compartment) aligned above the actuation chamber (bottom compartment) separated by the PDMS membrane. b) Mechanisms used to stretch and to c) electrically stimulate the microtissues. Adapted from [6].

\section{B. Cell culture and viability analysis}

Neonatal rat cardiomyocytes (NRCMs) were embedded at high density $\left(1 \times 10^{6} \mathrm{cell} / \mathrm{s} / \mathrm{ml}\right)$ in fibrin gel, injected in the central channel of the culture chamber and cultured for 5 days under 
mechanical deformation $(10 \%$ strain, $1 \mathrm{~Hz})$ or in static conditions. In detail, the actuation compartment was filled with PBS and a pressure of $0.5 \mathrm{~atm}$ was cyclically applied. The pressure caused the membrane to deflect till the pillars are reached, so to stretch the confined microtissue (Fig.1b), as previously described [6]. Furthermore, electrical stimulation (biphasic stimulation at $1 \mathrm{~Hz}$ with an electric field at $5 \mathrm{~V} / \mathrm{cm}$ ) was performed for 2 days on microtissues cultured in static condition for 3 days, by inserting two stainless steel wires within the lateral media channels, to have electrodes parallels to the microtissues (Fig.1c).

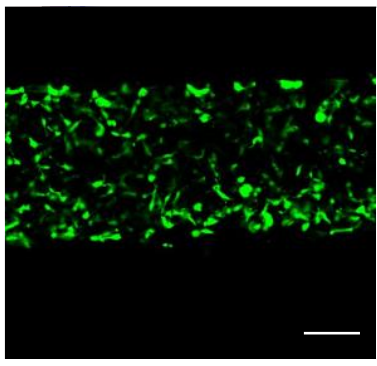

Control (static)

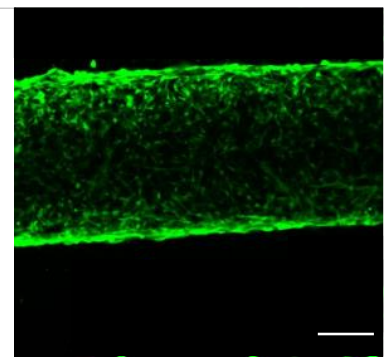

Mechanically actuated
Fig. 2. Live cells distribution in control (left) and mechanically actuated (right) micro-channels. Scale bar $100 \mu \mathrm{m}$.

Cells stained with Calcein AM (Fig. 2) demonstrates that the distribution of live cells is non uniform. Their concentration increases close to the feeding channels. The directionality diagrams are shown in Figure 3 (zero angle corresponds to the cells elongated in the direction perpendicular to $x$ axes). It can be seen that in the middle of the gel the cells are distributed more chaotically in comparison with the areas near the feeding channels, especially for mechanically actuated conditions. The area between pillars was divided into three or five equal parts in order to calculate cell concentrations and directionality.
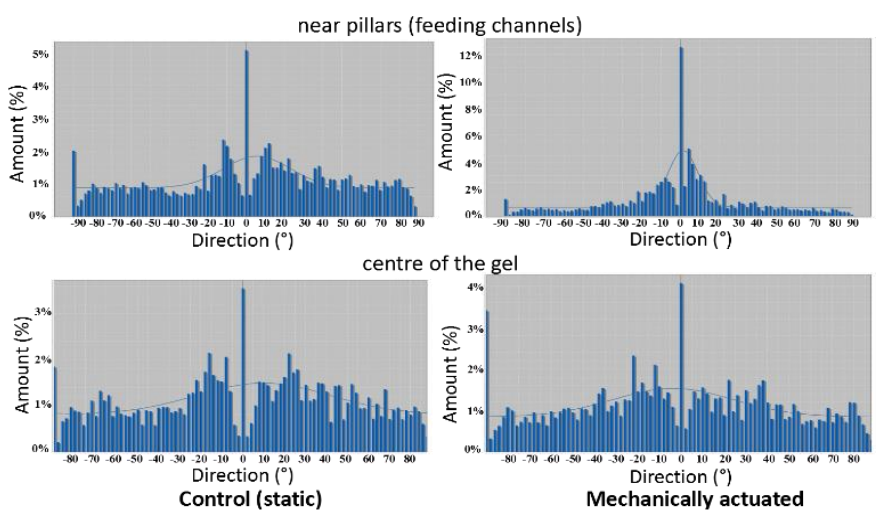

Fig. 3. Live cells directionality diagrams (amount of cells in percentage versus angle between the cell axis and the horizontal direction perpendicular to axis $x$ )in control (left) and mechanically actuated (right) micro-channels obtained with the use of ImageJ software.

Also within electrically stimulated constructs, cells distribution resulted not uniform, with cells concentrating near the pillars, as evidenced by bright field images (Fig. 4). This finding was confirmed by analyzing through ImageJ the intensity and directionality of the cells within the gel (Fig. 5).
These facts are at the base of our interest to investigate the distribution of the nutrients concentration in different zones of the chip as a reason of non-uniformity of the cells concentration and directionality.

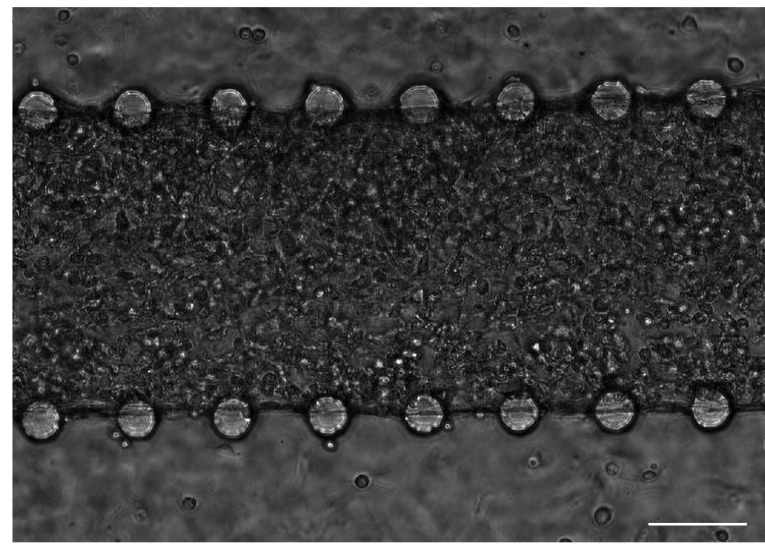

Fig. 4. Bright field image evidencing cell distribution within microtissues electricallystimulated for 2 days. Scale bar $100 \mu \mathrm{m}$.

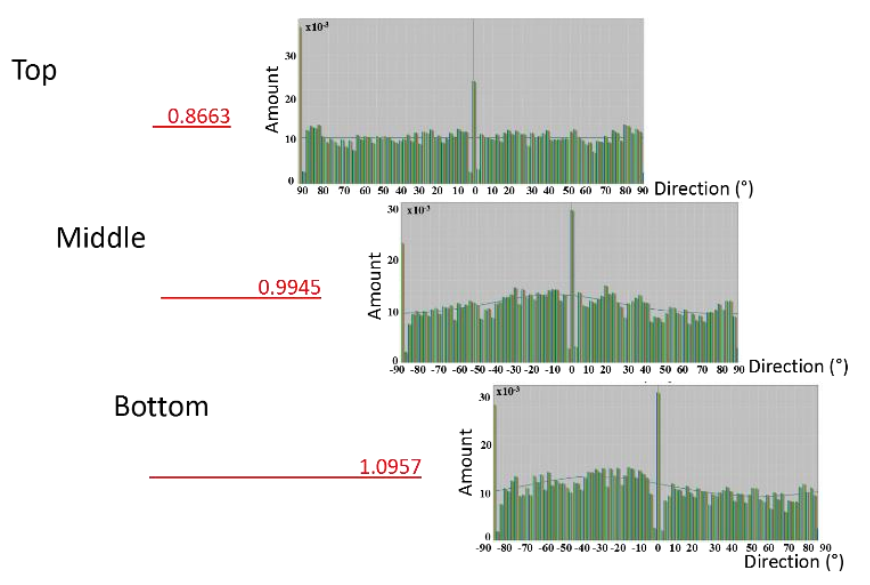

Fig. 5. Intensity and directionality (similar to the shown in Fig.3) diagrams for electrically stimulated cardiac microtissues obtained with the use of ImageJ software. Dimensionless intensities of signal (based on the value averaged on all the area between pillars) are shown in red.

\section{One-dimensional equation fot a nutrient or a waste concentration}

To investigate the nutrient/waste concentration $c(x, y, z, t)$, the diffusion equation can be used [9]:

$$
\frac{\partial c}{\partial t}-\nabla[D(x, y, z, t, c) \nabla c]=q(x, y, z, t, c)
$$

One-dimensional case with the constant diffusion coefficient $D\left(\mathrm{~m}^{2} / \mathrm{s}\right)$ and eating rate $q\left(\mathrm{~mol} /\left(\mathrm{m}^{3} \mathrm{~s}\right)\right)$ yields

$$
\frac{\partial \bar{c}}{\partial \bar{t}}-\frac{D T}{L^{2}} \frac{\partial^{2} \bar{c}}{\partial \bar{x}^{2}}=\frac{q T}{c_{0}}, \quad \bar{c}=\frac{c}{c_{0}}, \quad \bar{t}=\frac{t}{T}, \quad \bar{x}=\frac{x}{L}
$$

where $c_{0}$ is the initial concentration of the nutrient (or waste), $L$ is the typical size of the micro-channel, $T$ is the typical time 
of the process. Dimensionless parameter $D T / L^{2}$ can achieve very high values in micro-channels due to their small dimensions. E.g., with the values $D=10^{-9} \mathrm{~m}^{2} / \mathrm{s}, L=10^{-4} \mathrm{~m}, \mathrm{~T}=$ $1000 \mathrm{~s}$, the parameter $D T / L^{2}=100$. It means that time derivatives can be neglected and we can find the quasi-steady solution of (2)

$$
\frac{d^{2} \bar{c}}{d \bar{x}^{2}}=-\bar{q}=-\frac{q L^{2}}{D c_{0}}
$$

or

$$
\bar{c}(\bar{x}, \bar{t})=-0.5 \overline{q x}^{2}+b(\bar{t}) \bar{x}+a(\bar{t})
$$

where functions $a(\bar{t})$ and $b(\bar{t})$ must be found from the boundary and initial conditions.

\section{RESULTS AND DISCUSSION}

If the cells don't eat and there is no nutrient flux on the boundaries, eq. (4) yield the trivial solution

$$
\bar{c}=a
$$

Thus, we can expect to have constant concentrations of nutrients in the lateral channels after 5-10 minutes after their filling with solutions. If the cells located in gel do not eat, the same concentration will be also in the gel. The value of this equilibrium concentration can be obtained with the use of trivial mass balance (if we consider the symmetric problem with two lateral feeding channels of the same width $l_{1}$ filled at the beginning with the nutrient with concentration $c_{0}$ ):

$$
\bar{c}=a=\frac{2 \bar{l}_{1}}{2 \bar{l}_{1}+2}, \quad \bar{l}_{1}=\frac{l_{1}}{L}
$$

Here $L$ is the half of the gel width.

The same symmetric problem with eating cells and the origin of coordinate $x$ located in the middle of the gel must yield

$$
\left.\frac{\partial \bar{c}}{\partial \bar{x}}\right|_{\bar{x}=0}=0
$$

Then it follows from (4) and (6): $b=0$,

$$
\bar{c}(\bar{x}, \bar{t})=-0.5 \overline{q x}^{2}+a(\bar{t})
$$

Since in the lateral channels the concentration is uniform and equal to some unknown function $\bar{c}_{1}(\bar{t})$, the solution (7) can be written as follows:

$$
\bar{c}(\bar{x}, \bar{t})=-0.5 \bar{q}\left(\bar{x}^{2}-1\right)+\bar{c}_{1}(\bar{t})
$$

in order to have a continuous concentration distribution at $\bar{x}=1$.

Function (8) has a minimum at $\bar{x}=0$ (since in the case of eating, $\bar{q}<0$ ) and it is possible to have a zero value of concentration at this point in the critical moment of time which can be calculated from the equation:

$$
\bar{c}_{1}\left(\bar{t}_{c r}\right)=-0.5 \bar{q}
$$

For $\bar{t} \leq \bar{t}_{c r}$ function $\bar{c}_{1}(\bar{t})$ can be found from the simple mass balance:

$$
\int_{0}^{1} \bar{c}(\bar{x}, \bar{t}) d x+\bar{c}_{1}(\bar{t}) \bar{l}_{1}=1 \cdot \bar{l}_{1}+\frac{q t}{c_{0}}
$$

Putting in (10) formula (8), we can obtain

$$
\bar{c}_{1}(\bar{t})=\frac{\overline{l_{1}}+\bar{q}\left(\bar{t} \frac{D T}{L^{2}}-\frac{1}{3}\right)}{1+\bar{l}_{1}}
$$

With the use of (11) and (9) we can obtain the value of $\bar{t}_{c r}$

$$
\bar{t}_{c r}=-\frac{L^{2}}{D T}\left(\frac{\bar{l}_{1}}{\bar{q}}+\frac{\bar{l}_{1}}{2}+\frac{1}{6}\right)
$$

Since this value must be positive, the eating rate must be limited for this quasi-steady solution by the value:

$$
\bar{q}>-\frac{6 \bar{l}_{1}}{3 \bar{l}_{1}+1}
$$

Eating cells produce some waste. If its rate $w>0$ is uniform and time independent, its concentration in the quasi-steady case can be calculated with the equation similar to (8)

$$
\bar{c}_{W}(\bar{x}, \bar{t})=-0.5 \bar{w}\left(\bar{x}^{2}-1\right)+\bar{c}_{1 W}(\bar{t}), \quad \bar{w}=\frac{w L^{2}}{D_{W} c_{0}}
$$

Function $\bar{c}_{1 W}(\bar{t})$ can be found from the simple mass balance:

$$
\int_{0}^{1} \bar{c}_{W}(\bar{x}, \bar{t}) d x+\bar{c}_{1 W}(\bar{t}) \bar{l}_{1}=\frac{w t}{c_{0}}
$$




$$
\bar{c}_{1 W}(\bar{t})=\frac{\bar{w}\left(\bar{t} \frac{D_{w} T}{L^{2}}-\frac{1}{3}\right)}{1+\bar{l}_{1}}
$$

Since the waste concentration cannot be negative, the quasisteady solution (14), (16) is applicable only when

$$
\bar{t} \geq \frac{L^{2}}{3 D_{w} T}
$$

The results of calculations with the use of solutions (8), (11) and (14), (16) for $D=D_{w}=2 \cdot 10^{-9} \mathrm{~m}^{2} / \mathrm{s}, L=1.5 \cdot 10^{-4} \mathrm{~m}, T=$ one day $=8.64 \cdot 10^{4} \mathrm{~s}, \bar{l}_{I}=4$ are presented in Fig. 6 for the case of intensive eating/wasting rate. It can be seen, that the nutrient concentration can be much lower in the middle of the gel (far from the feeding channels). Accordingly, in the middle of the gel the concentration of the waste is the highest. Thus, the regions near the feeding cannels can be favorable for cells, and they can migrate closer to nutrients.

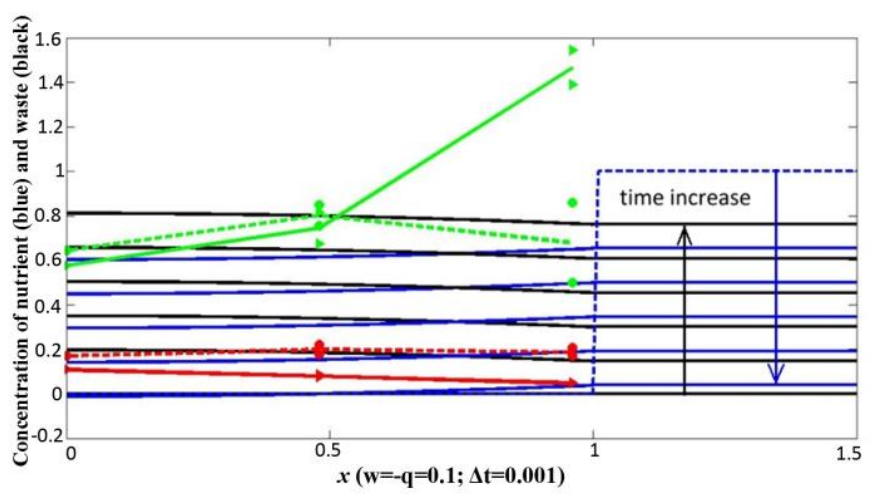

Fig. 6.Dimensionless concentrations of nutrients (blue lines) and waste (black lines) in gel and lateral feeding channel (coordinate $x$ is shown in Fig.1) for $\bar{w}=-\bar{q}=0.1$. The time interval between lines is 0.001. Experimental data for live (green lines and markers) and dead cells (red lines and markers) in a chip from [6]. Solid lines and triangles correspond to the mechanically actuated cases. Dashed lines and circles correspond to control static conditions.

The experimental data (from [6]) for dimensionless cells concentration (based on the average concentration of live cells) are also presented in Fig. 6. It can be seen that the concentration of live cells increases rapidly near the feeding channels, especially for the mechanically actuated cases. Dead cells are distributed uniformly. In addition, their concentration decreases for the mechanically actuated cases.

The calculations of the critical time (according to the formula (12)) are shown in Fig. 7. It can be seen that increasing the eating rate the critical time decreases. The critical time increases linearly with the increasing of the feeding channel width.

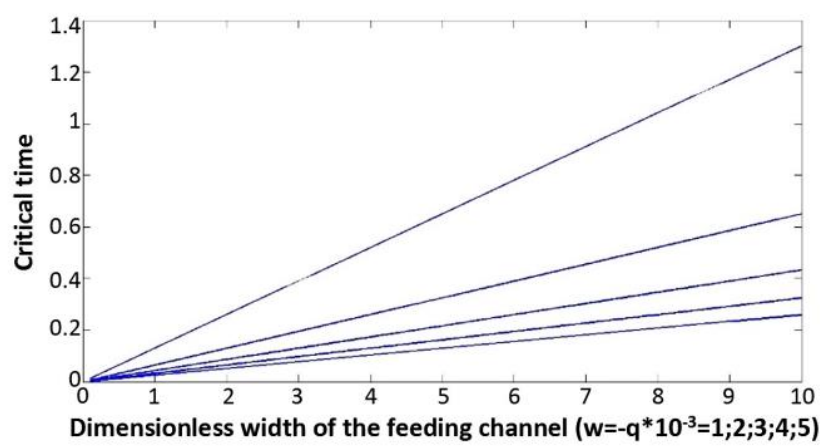

Fig. 7. Dimensionless critical time versus dimensionless width of the feeding channel for different feeding/wasting rates $\bar{w}=-\bar{q}=0.001 ; 0.002 ; 0.003$; 0.004; 0.005 from up to down lines respectively.

\section{CONCLUSIONS}

In this work, we have shown that the nutrients and waste concentration distribution can be non-uniform through the chip and can cause different environmental conditions, influencing cell culture. Obtained analytical formulas for the nutrient/waste concentration and the critical time could be useful for analysis of the cell behavior under electrical and/or mechanical stimulation and can improve the method and protocols of cell cultivation and chip design. In order to have precise estimations of the concentration distribution, non-steady 2D and 3D models must be used.

\section{ACKNOWLEDGMENT}

The study was supported by EU-financed Horizon-2020 project AMMODIT (Grant Number MSCA-RISE 645672).

\section{REFERENCES}

S. N. Bhatia and D. E. Ingber, "Microfluidic organs-on-chips," Nature Biotechnology, vol. 32, no. 8. pp. 760-772, 2014.

[2] K. Gupta et al., "Lab-on-a-chip devices as an emerging platform for stem cell biology," Lab Chip, vol. 10, no. 16, p. 2019, 2010.

[3] A. Pavesi, G. Adriani, M. Rasponi, I. K. Zervantonakis, G. B. Fiore, and R. D. Kamm, "Controlled electromechanical cell stimulation ona-chip," Sci. Rep., vol. 5, 2015.

[4] A. a Werdich et al., "A microfluidic device to confine a single cardiac myocyte in a sub-nanoliter volume on planar microelectrodes for extracellular potential recordings.," Lab Chip, vol. 4, pp. 357-362, 2004

[5] D. Huh, B. D. Matthews, A. Mammoto, M. Montoya-Zavala, H. Yuan Hsin, and D. E. Ingber, "Reconstituting organ-level lung functions on a chip," Science (80-. )., vol. 328, no. 5986, pp. 1662-1668, 2010.

[6] A. Marsano et al., "Beating heart on a chip: a novel microfluidic platform to generate functional 3D cardiac microtissues," Lab Chip, vol. 16, no. 3, pp. 599-610, 2016.

[7] R. J. McMurtrey, "Analytic Models of Oxygen and Nutrient Diffusion, Metabolism Dynamics, and Architecture Optimization in Three-Dimensional Tissue Constructs with Applications and Insights in Cerebral Organoids," Tissue Eng. Part C Methods, vol. 22, no. 3, pp. 221-249, 2016.

[8] M. C. Kim, R. H. W. Lam, T. Thorsen, and H. H. Asada, "Mathematical analysis of oxygen transfer through polydimethylsiloxane membrane between double layers of cell culture channel and gas chamber in microfluidic oxygenator," Microfluid. Nanofluidics, vol. 15, no. 3, pp. 285-296, 2013.

[9] Loitsyanskiy, L.G. Mechanics of Liquids and Gases, Begell House, New York and Wallingford, $6^{\text {th }}$ ed., 1995 